\section{Effect of Rosuvastatin on the Renal Cortex of Adult Male Albino Rats and the Possible Protective Effect of Co-enzyme Q 10 (Light and Electron Microscopic Study)}

* Corresponding Author:

Mohammed Almandouh

Mohamed2010808@yahoo.com

Received for publication August

5, 2020; accepted September 2,

2020; published online

September 2, 2020.

Copyright 2020 The Authors published by Al-Azhar University, Faculty of Medicine, Cairo, Egypt. All rights reserved. This an openaccess article distributed under the legal terms, where it is permissible to download and share the work provided it is properly cited. The work cannot be changed in any way or used commercially.

doi: 10.21608/aimj.2020.34957.1272

1 Anatomy and Embryology

Department, Faculty of

Medicine, Al-Azhar University,

Cairo, Egypt.

\begin{abstract}
Background: Rosuvastatin can decrease the low-density lipoprotein cholesterol to optimal goals. Adverse effects of rosuvastatin involve the kidneys. Multiple studies explained that these side effects are due to reducing coenzyme Q ten (Q10) levels.

Objective: to throw light upon the histological changes in the renal cortex of albino rats treated with rosuvastatin and explore the impact of CoQ10 co-treatment.

Methods: The study was done at the Pharmacology Department lab., Faculty of Medicine, Al-Azhar University between August and October 2019. Forty adult albino rats were used in the study as follows: Group I: formed of 8 rats, given $1 \mathrm{ml}$ distilled water/day for one month orally; Group II: formed of 8 rats, given 1.8 milligrams/Kilogram/day of CoQ10 for one month orally; Group III: formed of 8 rats, given 0.72 milligrams/Kilogram/day of rosuvastatin for one month orally; Group IV: formed of 8 rats, given 0.72 milligrams/Kilogram/day of rosuvastatin and 1.8 milligrams/Kilogram/day of CoQ10 for one month orally; Group V: formed of 8 rats, received the same dose of rosuvastatin as in group III then the rats were sacrificed two weeks later.

Results: In group III, there were severe histo-pathological changes in the renal corpuscles and tubules. Also, the biochemical results (serum urea and creatinine) were deteriorated. In groups V and IV, the biochemical results were improved significantly. The histological integrity of the renal cortex appeared to return to the normal features found in the control group I.

Conclusion: This study revealed that CoQ10 might have a protective role against rosuvastatin nephrotoxicity.

Keywords: Rosuvastatin; CoQ10; rats; renal cortex

Authorship: All authors have a substantial contribution to the article.

Disclosure: The authors have no financial interest to declare in relation to the content of this article. The Article Processing Charge was paid for by the authors.
\end{abstract}

\section{INTRODUCTION}

The renal cortex is formed of glomeruli, proximal convoluted tubules (PCT), cortical portions of loops of Henle, distal convoluted tubules (DCT), and cortical portions of collecting ducts. ${ }^{1}$

Statins are the best-tolerated drugs for the treatment of conditions associated with dyslipidemia. They reduce the risk of initial and recurrent cardiovascular events. Rosuvastatin can decrease LDL cholesterol to its normal range and increase HDL cholesterol levels. ${ }^{2}$ Rosuvastatin has many adverse effects on kidneys, liver, and muscles. These side effects are due to reducing serum coenzyme Q10 levels. ${ }^{3}$
Coenzyme Q10 is usually synthesized through some reactions from mevalonic acid. Mevalonic acid production is inhibited by hydroxyl-methylglutaryl coenzyme A reductase inhibitors. Most of the serum Coenzyme Q10 is bounded to the (LDL) cholesterol. ${ }^{4}$

This study aims to throw light upon the histological changes in the renal cortex of albino rats treated with rosuvastatin and explore the impact of CoQ10 cotreatment.

\section{MATERIAL AND METHODS}

Animals:

The study was done at the pharmacology department laboratory of Al-Azhar Faculty of Medicine, Cairo, between August and October 2019. 
Experimental design:

Fourty albino rats were divided equally into 5 groups (each group formed of 8 rats) as follows:

Group I: (Control rats) received $1 \mathrm{ml}$ isotonic saline /day orally for four weeks. ${ }^{5}$

Group II: (CoQ10 rats) received 1.8 milligrams/Kilogram/day of CoQ10 orally for four weeks. $^{6}$

Group III: (Rosuvastatin rats) received 0.72 milligrams/Kilogram /day of rosuvastatin orally for four weeks. ${ }^{7}$

Group IV: (Rosuvastatin and CoQ10 rats) received 0.72 milligrams/Kilogram/day of rosuvastatin and 1.8 milligrams/Kilogram/day of CoQ10 for four weeks orally. $^{6}$

Group V: (Withdrawal rats) received the same dose of rosuvastatin for four weeks then the rats were sacrificed after the subsequent two weeks. ${ }^{8}$

\section{Chemicals:}

Preparation of co-enzyme Q10: "Co-enzyme Q10" capsule $200 \mathrm{mg}$. Every capsule was dissolved in 200 $\mathrm{ml}$ distilled water so every $1 \mathrm{ml}$ contained $1 \mathrm{mg}$ Coenzyme Q10.

Preparation of rosuvastatin: "Rosuvastatin" tablet 40 $\mathrm{mg}$. Every tablet was dissolved in $40 \mathrm{ml}$ distilled water so every $1 \mathrm{ml}$ contained $1 \mathrm{mg}$ of rosuvastatin. The two drugs were purchased from Faculty of Pharmacy, ALAzhar University, Egypt

At the assigned time, rats were anesthetized using ether inhalation. Blood samples were taken through retro-orbital puncture for measurement of urea and creatinine. The kidneys were carefully dissected. One specimen was prepared and stained with hematoxylin and eosin (H\&E) and PAS stains for light microscopic examination. ${ }^{9}$ The other specimen was prepared and stained with a double staining technique of uranyl acetate, followed by lead citrate solutions for electron microscopic examination. ${ }^{10}$

\section{RESULTS}

Light microscopic findings:

In groups I and group II, the renal cortex consisted of two main parts. It was formed of the renal corpuscles integrated with the renal tubules and minimal interstitium containing peritubular capillaries. The glomerulus was surrounded by the two layers of Bowman's capsule appeared as the main components of the renal corpuscle. Bowman's space appeared separating the parietal layer of Bowman's capsule from the visceral layer. The proximal (PCT) and distal convoluted tubules (DCT) were the main cortical tubules. The nuclei of the PCT and DCT were spherical and centrally located in addition to their cuboidal epithelial lining. The lumen of the DCT was wider and regular due to the absence of the brush border (BB) in the distal tubules (Figures 1,2).

In rosuvastatin-treated rats, most of the renal corpuscles showed small-sized distorted hypercellular glomeruli. The Bowman's spaces (BS) appeared widened or obliterated. The proximal tubules showed markedly edematous epithelium with severe apoptotic changes. The brush border (BB) of the proximal convoluted tubules (PCT) was partially lost. The distal convoluted tubules (DCT) appeared with intraluminal debris and severely congested interstitial blood vessels. Intratubular vacuolation with granular casts was seen. Congestion of blood vessels with areas of hemorrhage was seen in the renal cortex (Figure 3). In groups IV \&V, the histological architecture of the glomerulus, PCT, and DCT returned to the normal histology detected in the control group (Figure 4,5).

Electron microscopic findings:

Groups I and II revealed the normal histology of the glomerular capillaries which was formed of three layers arranged from outside as follows: podocytes, glomerular basement membrane (GBM), and fenestrated endothelium.

The PCT showed large nuclei which were spherical, basally located, and euchromatic with prominent nucleoli. The cytoplasm showed mitochondria with cristae, dense long micro-villi, normal (BB), and normal basal lamina.

The DCT showed cells with few short microvilli, round centrally located nuclei with central nucleoli and condensed chromatin in the periphery, normal mitochondria, and basal infoldings (Figures 6,7).

The podocytes showed two cytoplasmic processes. The primary and secondary foot processes of the podocyte appear close to the GBM. The GBM was uniform in thickness (Figure 8).

Group III rats showed loss of the glomerular architecture with an irregular basement membrane, podocyte with an irregular nucleus, marked effacement of foot processes, and very narrow filtration slits.

The PCT in group III showed wide lumen with few long microvilli, basally located heterochromatic markedly pyknotic nuclei with prominent nucleoli, swollen mitochondria, scattered cytoplasmic vacuoles, and thickened basal lamina.

The DCT in group III showed few short microvilli, centrally located heterochromatic markedly pyknotic nuclei with centrally located nucleoli, swollen mitochondria, and intra-luminal debris obliterating most of the lumen (Figures 9-11).

Groups IV and V showed the normal structure of the glomerular capillaries, PCT, and DCT (Figures 1214). 


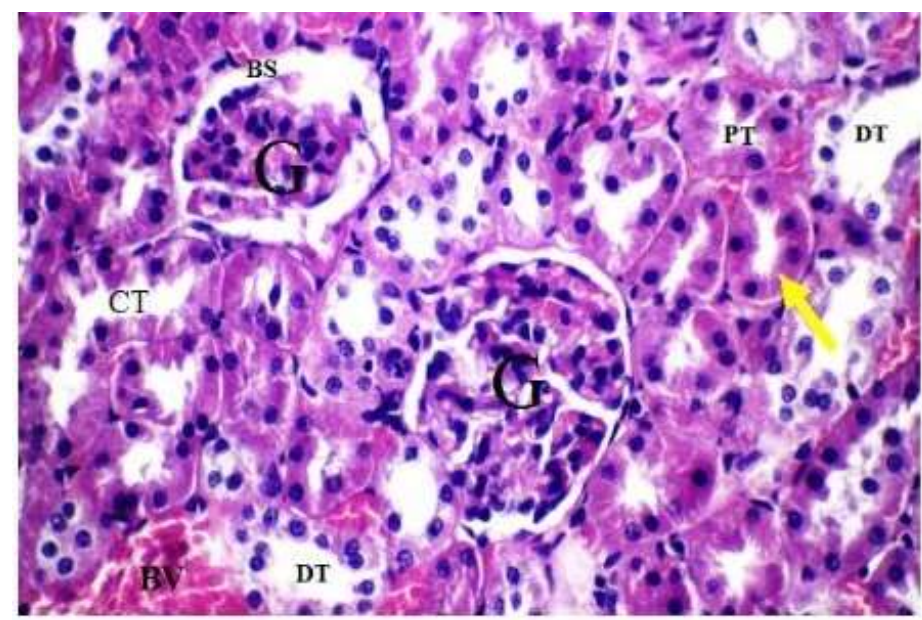

Fig.1: A photomicrograph of Hematoxylin and Eosin section (group I), showing rounded glomeruli (G), each is surrounded by the two layers of Bowman's capsule with Bowman's space (BS). In addition to glomeruli, the cortex consisted mainly of PCT (PT), and DCT (DT). The PCT has highly defined brush borders (arrowhead) with a normal basement membrane. (H\&E. X360).

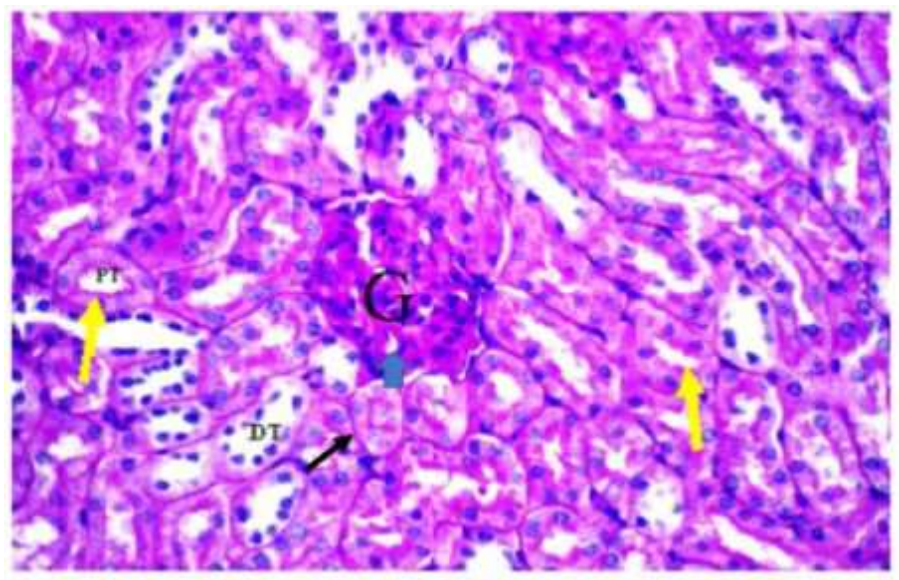

Fig. 2: A photomicrograph of Hematoxylin and Eosin section (group II), showing renal corpuscles formed of a glomerulus $(\mathrm{G})$ in addition to the PCT (PT) and DCT (DT). Proximal tubules show normal epithelial lining with normal brush borders (yellow arrow), and normal BM (black arrows) (H\&E. X360).

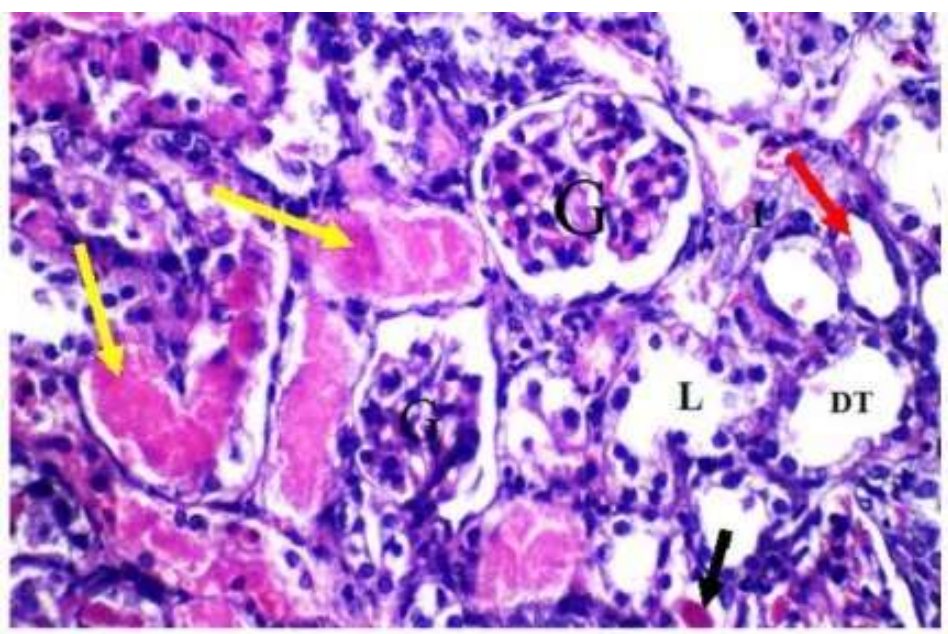

Fig. 3: A photomicrograph of Hematoxylin and eosin section (group III), showing small-sized distorted glomerulus (G) and average Bowman's space. The PCT shows massive cellular edema and dilated lumen with apoptosis of its epithelial lining (black arrow). The brush borders of the PCT are completely lost with intratubular debris (red arrows) and intra-tubular granular casts (yellow arrow) (H\&E. X360). 


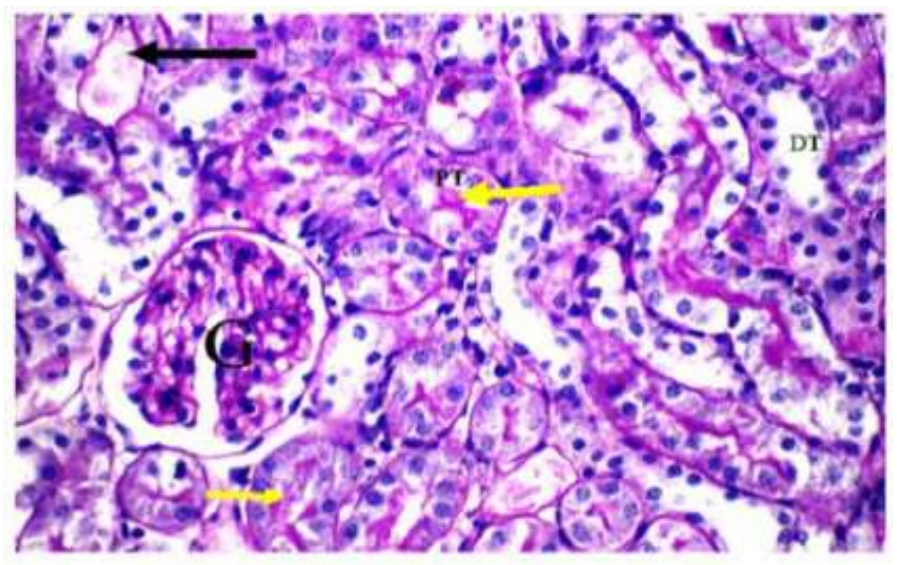

Fig. 4: A photomicrograph of PAS section (group IV) showing normal glomerulus (G), the brush borders of the PCT appear normal (yellow arrows) but he black arrow points a PCT with lost BB. Most of the DCT (DT) are normal (PAS. X360).

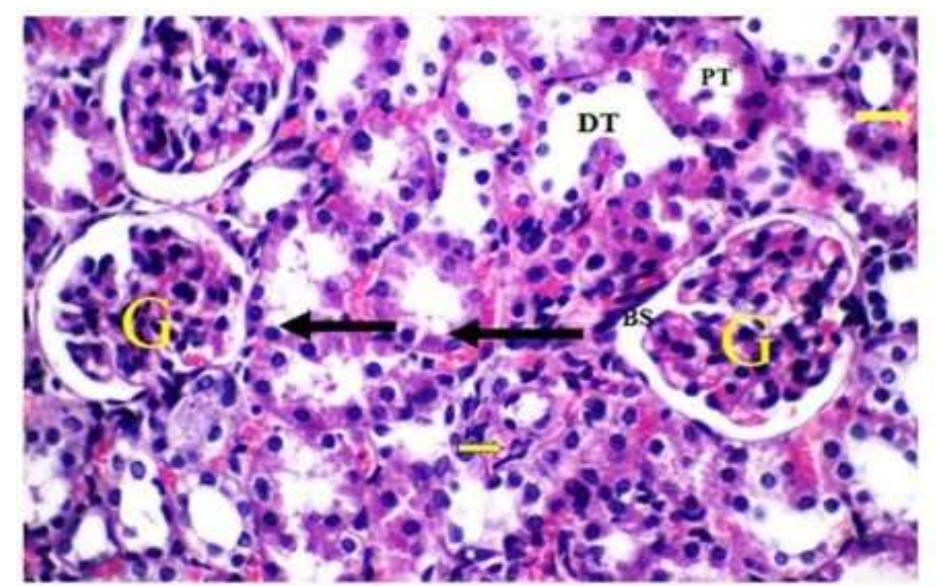

Fig. 5: A photomicrograph of Hematoxylin and eosin section (group V) showing the normal renal corpuscle formed of a glomerulus $(\mathrm{G})$ surrounded by the two layers of Bowman's capsule with Bowman's space. The PCT (PT) and DCT (DT) appear completely normal. Many tubules show normal epithelium (black arrows). Some capillaries appear dilatated and congested (yellow arrows) (H\&E. X360).

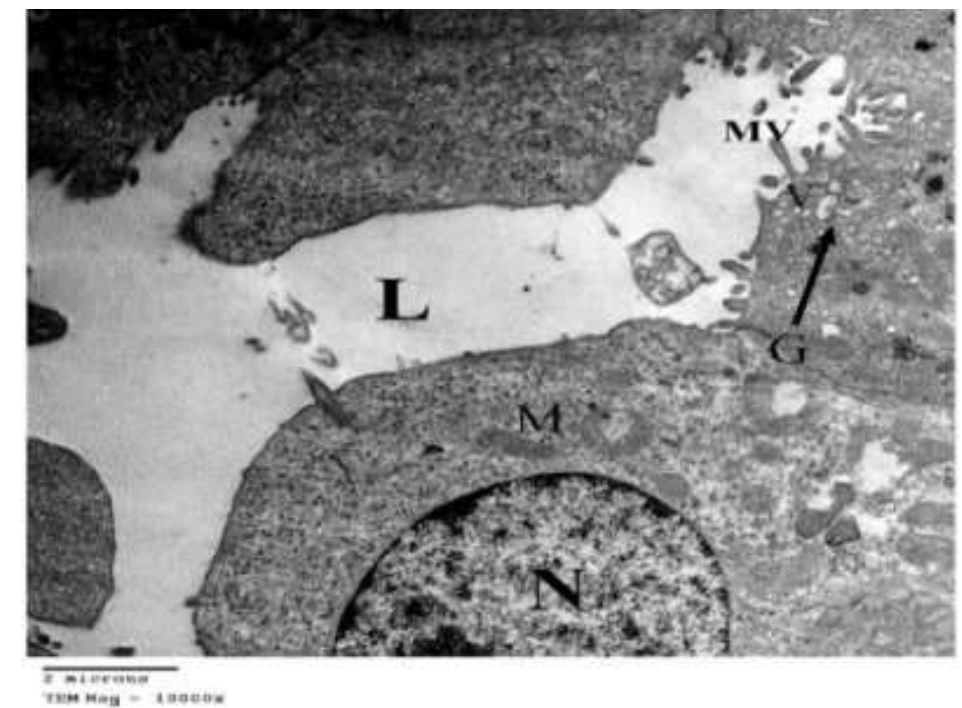

Fig. 6: An electron micrograph of the DCT (group I) revealing cells with few short microvilli (MV), Golgi apparatus $(\mathrm{G})$, round centrally located nuclei $(\mathrm{N})$ with central nucleoli and condensed chromatin in the periphery, normal mitochondria (M), and basal infoldings (TEM X10000). 


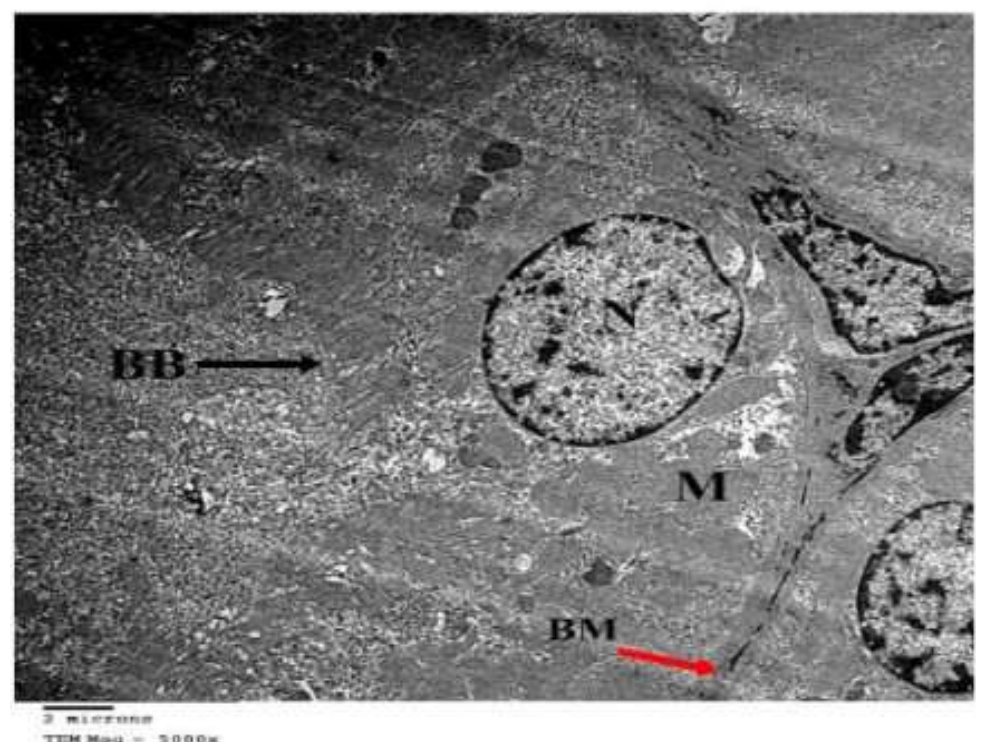

Fig. 7: An electron photomicrograph of the PCT (group I). It shows basally located rounded euchromatic nuclei (N) with prominent nucleoli (red arrow). The cytoplasm showing mitochondria with normal cristae (M), dense long microvilli forming a fantastic (BB), and intact basement membrane (BM) (TEM X5000).

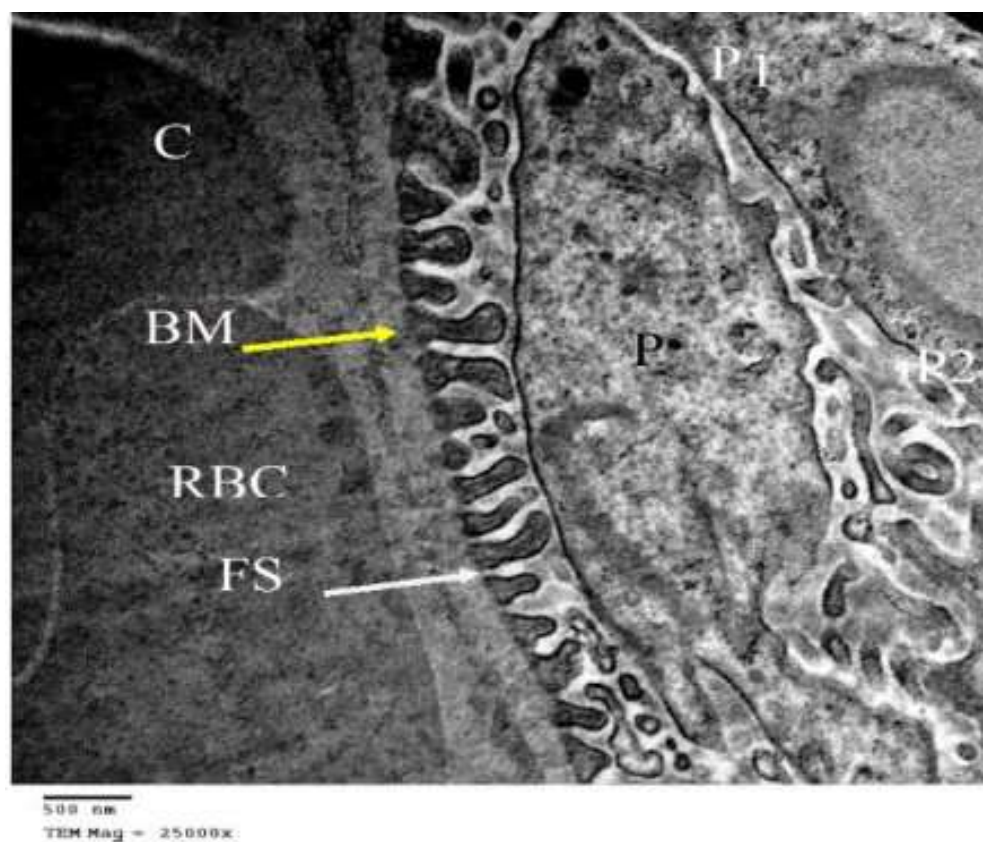

Fig. 8: An electron photomicrograph of the glomerulus (C) (group II) showing normal glomerulus with intact basement membrane (BM), Mesangial cell, normal Podocyte $(\mathrm{P})$, and its primary foot processes with patent filtration slits (FS). RBC= Red blood cell.(TEM X25000). 


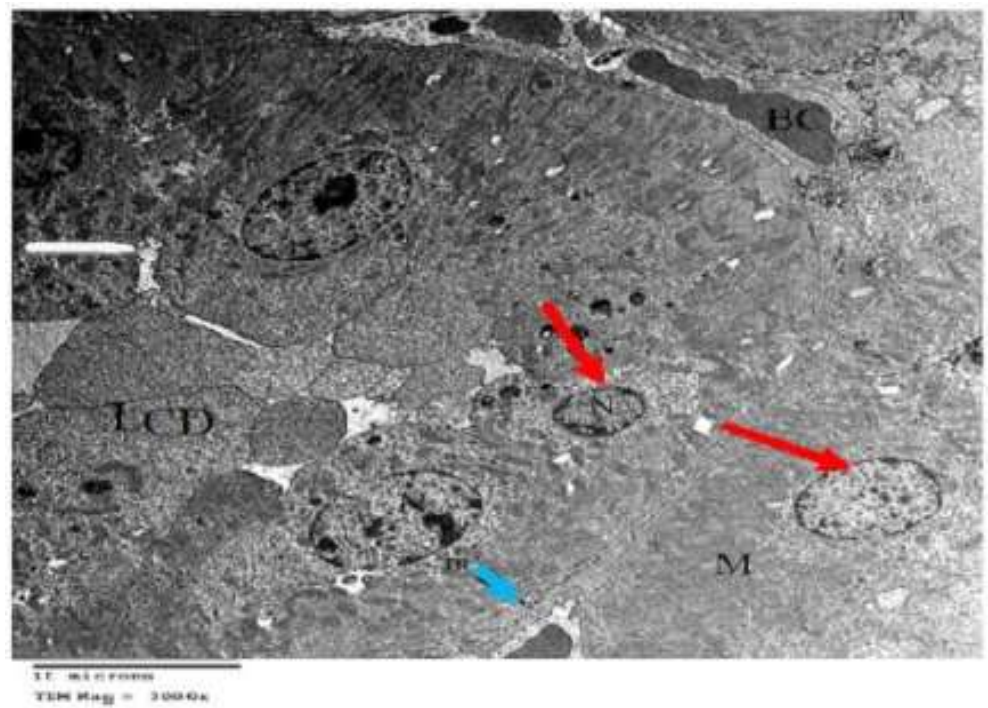

Fig. 9: An electron photomicrograph of the PCT (group III), showing shrunken nuclei with loss of their heterochromatin (red arrows). Intraluminal epithelial debris (LCD) can be seen. The apical microvilli are lost (white arrow). The basal lamina is interrupted (blue arrow). The mitochondria are swollen and edematous (M). $\mathrm{BC}=$ blood capillary (TEM X3000).

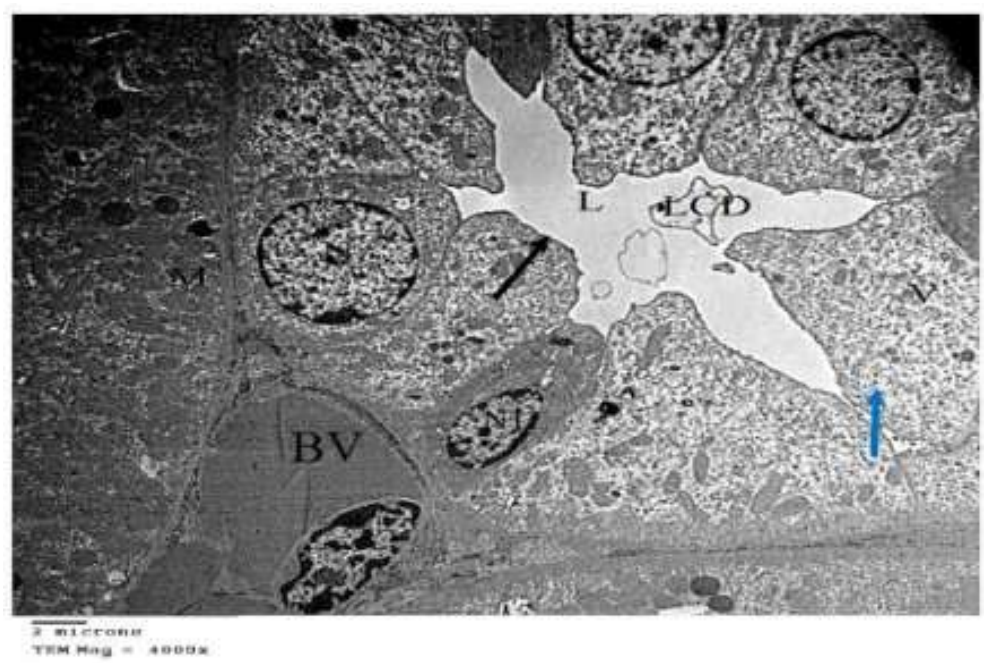

Fig. 10: An electron photomicrograph of the DCT (group III), showing intraluminal cellular debris (LCD) with a widened lumen (L). The apical microvilli are completely lost (Black arrow). Most of the nuclei are small-sized (N1) and irregular. Multiple vaculations are seen within the cytoplasm (Blue arrow). Note the swelling and edema of the mitochondria (M) with congestion and dilatation of blood vessels (BV) (TEM X4000). 


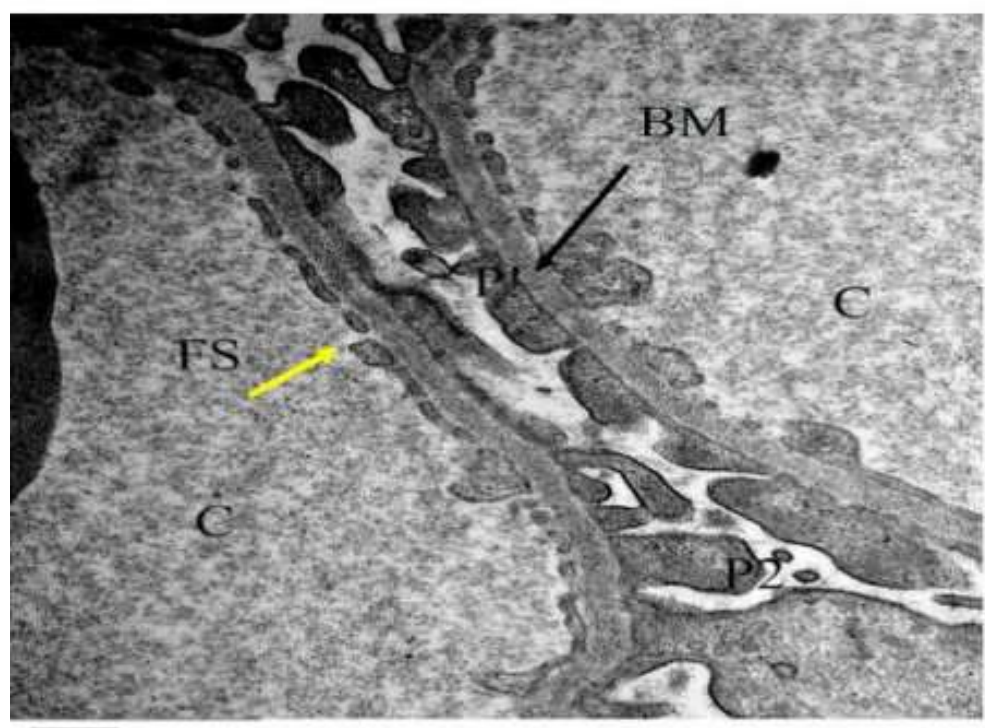

$\overline{\sin \sin }$

TEM Mag - $25068 x$

Fig. 11: An electron photomicrograph of the glomerulus (C) (group III), showing distorted glomerulus with irregular basement membrane (black arrow). The podocytes (P) show marked effacement of its processes (yellow arrow) with a very narrow filtration slit (FS) (TEM X25000).

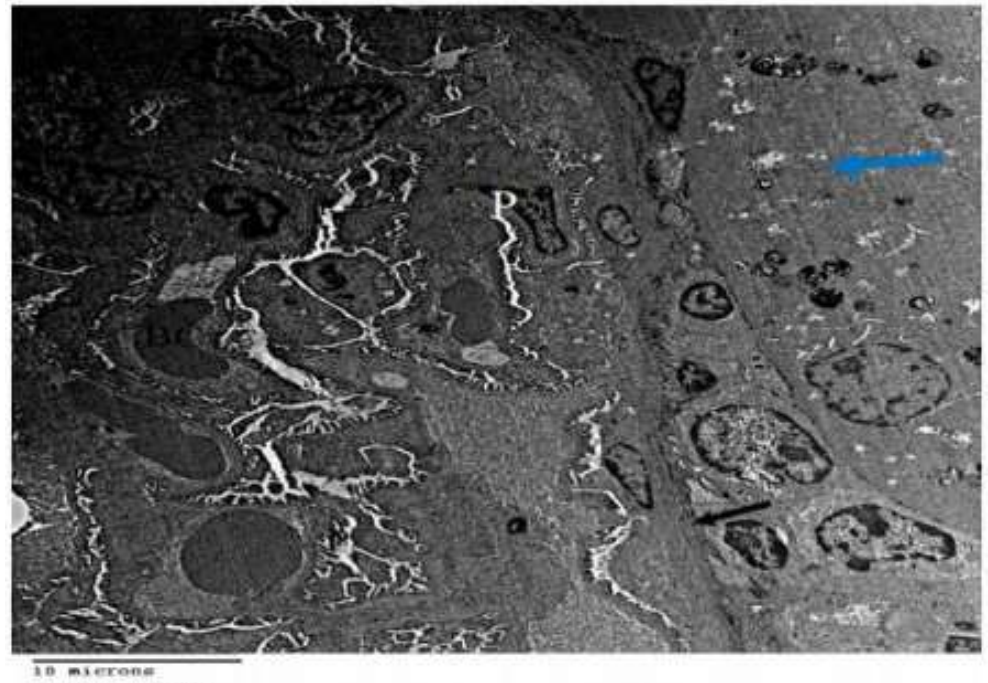

Fig. 12: An electron photomicrograph of the renal cortex (group IV), showing intact GBM, normal appearance of the proximal convoluted tubule (blue arrow), and normal podocyte with its processes $(\mathrm{P})$. The blood capillaries are mildly dilated (BC) (TEM X3000). 


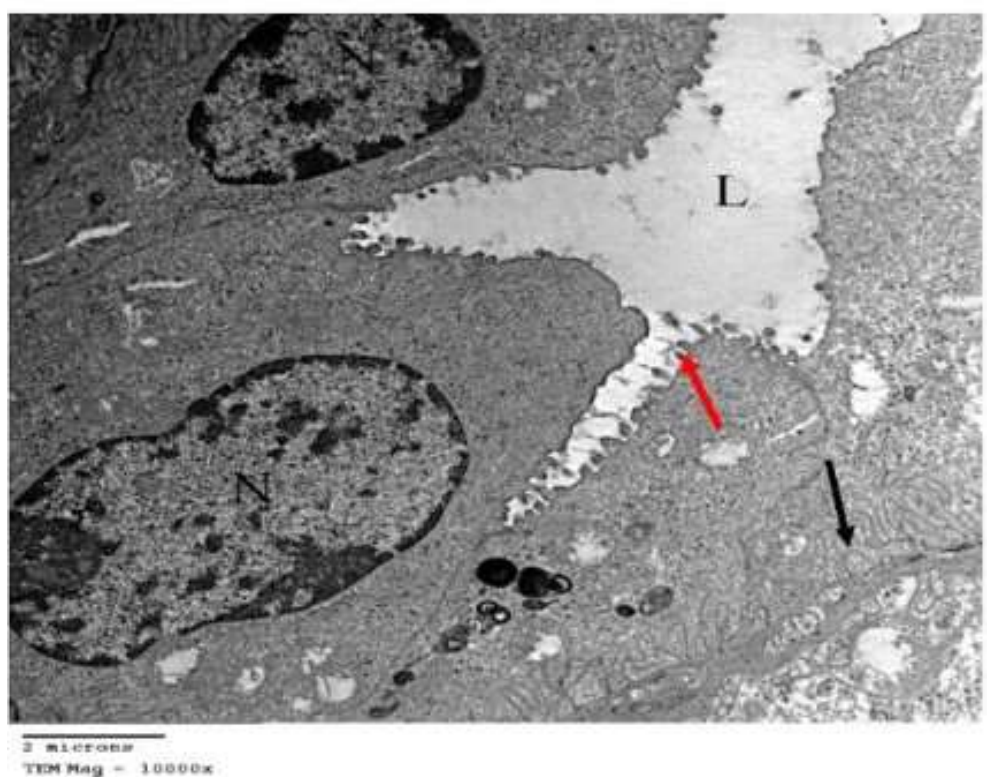

Fig. 13: An electron photomicrograph of the DCT (group IV) showing few short microvilli (red arrow), centrally located round nuclei $(\mathrm{N})$ with central nucleoli and condensed chromatin in the periphery, scattered vacuoles and basal infoldings (black arrow). L=lumen(TEM X10000).

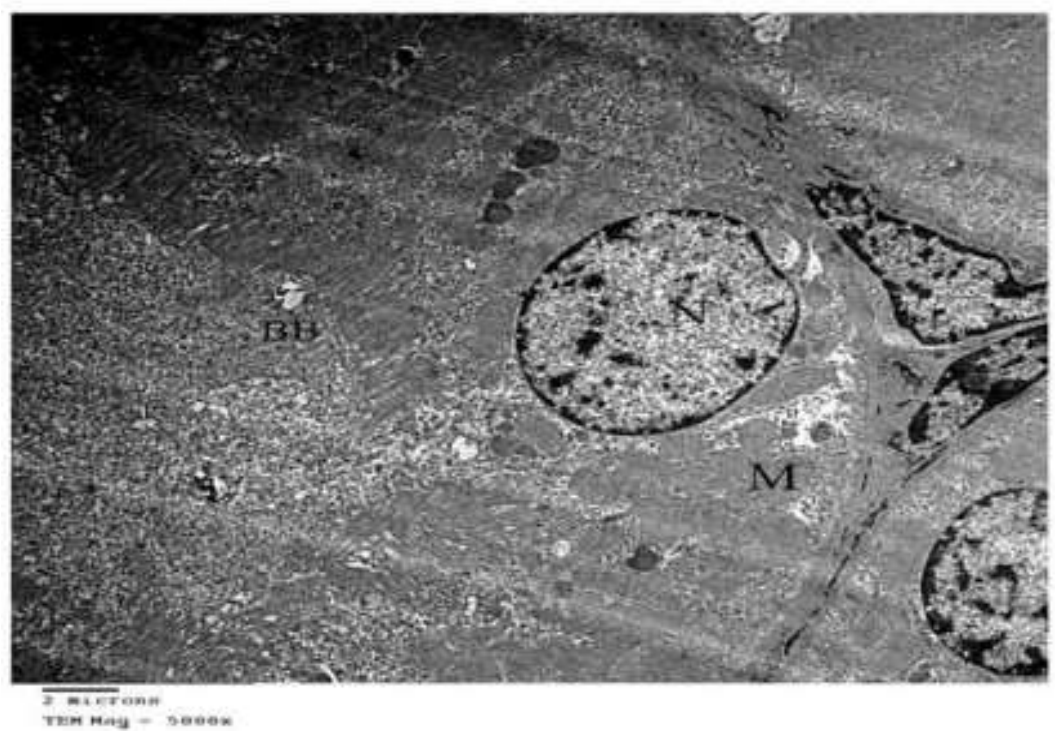

Fig. 14: An electron photomicrograph of the PCT (group V), showing spherical nucleus $(\mathrm{N})$, peripherally located chromatin, and normal (BB). The mitochondria appear normal (M) (TEM X5000).

\section{Biochemical results:}

By using the analysis of variance (ANOVA) and the least significant difference (LSD) tests, serum urea and creatinine showed a significant increase in the rosuvastatin-treated group. The biochemical results in groups IV and $\mathrm{V}$ were similar to that of group I (figure 15) (tables 1-3). 


\begin{tabular}{|c|c|c|}
\hline Group & & \\
\hline I & Mean urea & Std. Deviation \\
II & 21.963 & 0.5731 \\
III & 22.338 & 0.5605 \\
IV & 56.100 & 0.7653 \\
V & 21.538 & 0.5263 \\
\hline
\end{tabular}

Table 1: Comparison between the mean urea using the ANOVA test (analysis of variance).

\begin{tabular}{|c|c|c|}
\hline & & \\
\hline Group & Mean creatinine & Std. Deviation \\
\hline I & .6138 & 0.06760 \\
II & .5825 & 0.04400 \\
III & 2.5750 & 0.31960 \\
IV & 1.3100 & 0.03928 \\
V & 1.2650 & 0.04309 \\
\hline
\end{tabular}

Table 2: Comparison between the mean creatinine using the ANOVA test.

\begin{tabular}{|c|c|c|c|c|c|}
\hline Variable & Group & & Mean diff. & Std. error & Sig. \\
\hline Urea & Cont & $\begin{array}{l}\text { II } \\
\text { III } \\
\text { IV } \\
\text { V }\end{array}$ & $\begin{array}{c}-0.3750- \\
-34.137-^{*} \\
0.4250 \\
-0.3125-\end{array}$ & $\begin{array}{l}0.2958 \\
0.2958 \\
0.2958 \\
0.2958\end{array}$ & $\begin{array}{r}.213 \\
0.000 \\
0.160 \\
0.298\end{array}$ \\
\hline Creat & Cont & $\begin{array}{l}\text { II } \\
\text { III } \\
\text { IV } \\
\text { V }\end{array}$ & $\begin{array}{c}0.0000 \\
-10.937-^{*} \\
-1.5625- \\
-3.1250-\end{array}$ & $\begin{array}{l}2.8446 \\
2.8446 \\
2.8446 \\
2.8446\end{array}$ & $\begin{array}{l}1.000 \\
0.000 \\
0.586 \\
0.279\end{array}$ \\
\hline
\end{tabular}

Table 3: Comparison between the biochemical results in the different studied groups using the least significant difference (LSD). 


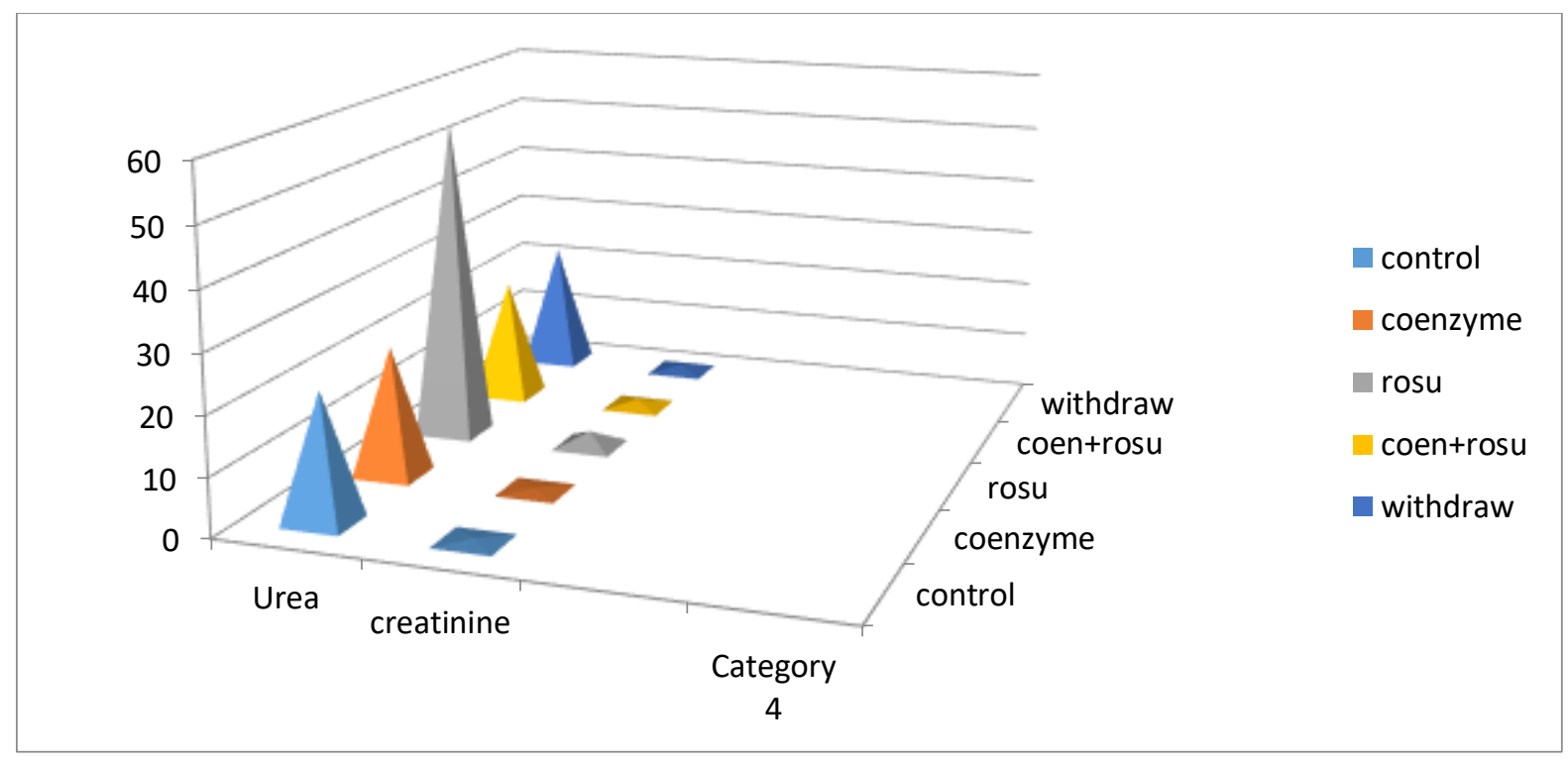

Fig. 15: Comparison between the mean urea and creatinine.

Statistical results:

By using one way ANOVA test, a significant increase in the rosuvastatin-treated group (p-value $<0.05$ was significant) was found. By LSD, a significant increase was found in group III. Statistical analysis showed no significant changes between the groups' IV and V as compared to group I.

\section{DISCUSSION}

In the current work, rosuvastatin and Co-enzyme Q10 were used for one month. The doses were 0.72 milligrams/Kilogram /day and 1.8 milligrams/Kilogram /day respectively. These doses were equivalent to normal human doses. ${ }^{11}$

In the current work, the examination of group I and group II revealed that the renal cortex consisted of two main parts. It was formed of the renal corpuscles integrated with the renal tubules and minimal interstitium containing peritubular capillaries. The glomerulus was surrounded by the two layers of Bowman's capsule. Bowman's space appeared separating the parietal layer of Bowman's capsule from the visceral layer. These results are in agreement with Rao et al. ${ }^{12}$

The PCT and DCT appeared as the main cortical tubules. The nuclei of the PCT and DCT were spherical and centrally located in addition to their cuboidal epithelial lining. The lumen of the DCT was wider and regular due to the absence of the brush border (BB) in the distal tubules. Similar results are reported by Ross and Pawlina. ${ }^{13}$

The rosuvastatin-treated group showed the renal corpuscles with congestion of the glomerular capillaries and increased irregularity of the parietal layer of Bowman's capsule. Some tubules showed hyaline casts in their lumen with abnormal dilatation of the others. These results are in concomitant with that reported by Ponnuswamy et al. ${ }^{14}$

In disagreement with Kostapanos et $\mathrm{al}^{15}$, rosuvastatin induced acute tubular necrosis and acute renal failure were due to the interstitial hemorrhage and congested peritubular capillaries.

In the current work, rosuvastatin induced abnormalities were in the form of glomerular sclerosis and swelling of the cells of the PCT and DCT with necrotic nuclei. These results are in accordance with Pisoni et al. ${ }^{16}$

In the current work, the renal cortex of group IV revealed normal renal corpuscles. Some tubular cells had a small darkly stained nucleus with the others showed intra-cytoplasmic vacuolation. These results are in agreement with Littarru. ${ }^{17}$

In the current work, group $\mathrm{V}$ revealed the normal histological architecture as group I. Also, the PCT and DCT showed normal histological structure. These results are in agreement with Galpern and Cudkowics. ${ }^{18}$

In the present work, PAS reaction in the renal cortex of group I was strongly positive in the $\mathrm{BM}$ of the glomerulus, PCT, and DCT. These results are coinciding with Yabuki et al. ${ }^{19}$

In the current study, in the rosuvastatin-treated group, PAS reaction appeared weak positive in the BM of the glomerular capillaries, Bowman's capsules (BC), PCT, and DCT. These results are in agreement with Thomas et al. $^{20}$ In groups IV\&V PAS reaction was strongly positive as the control group.

In the current work, the examination of the renal cortex of group I with an electron microscope showed the normal glomerular capillary wall. It was formed of three layers which were arranged from outside: podocytes, basement membrane (GBM), and fenestrated endothelium. Podocytes showed euchromatic nuclei with their primary and secondary foot processes. 
The rosuvastatin-treated group showed a distorted basement membrane with disorganized endothelium. Podocytes were seen with a heterochromatic nucleus, swollen mitochondria, and distorted foot processes. PCT cells were oval, rounded with irregular nuclei and heterochromatic clumps. Numerous swollen mitochondria and intracytoplasmic vacuolations were seen. Closely packed microvilli appeared at the luminal surface. DCT cells appeared with apoptotic nuclei. Mitochondria were seen with an irregular distribution. Irregular basal infoldings were also observed. Casts were found in the lumen. These results are in agreement with Brewer. ${ }^{21}$

Renal cell damage proven in our results is in disagreement with ${ }^{22}$ who demonstrated that proteinuria induced by rosuvastatin is likely to be associated with a statin provoked inhibition of low molecular weight protein reabsorption by the renal tubules.

Higher doses of rosuvastatin have been associated with cases of renal failure. Also, the co-administration of rosuvastatin with drugs that increase rosuvastatin blood levels may be deleterious for the kidney. These results are not coinciding with Rao et al. ${ }^{12}$

In the present work, the examination of the renal cortex of groups IV\&V with an electron microscope showed similar architecture as the control group.

In this study, the levels of serum urea and creatinine were similar in group I and group II. These renal function tests were significantly deteriorated in group III. In groups IV\&V they were improved towards the normal levels.

In conclusion, these results indicated that rosuvastatin which previously looked protecting from dyslipidemia and cardiovascular diseases may have disadvantages. So, we need to be very thoughtful about the haphazard usage of anti-cholesterolemic drugs as they may put us at risk for acute tubular necrosis.

Conflict of interest: No conflicts of interest were encountered.

\section{CONCLUSION}

In conclusion, these results indicated that rosuvastatin which previously looked protecting from dyslipidemia and cardiovascular diseases may have disadvantages. So, we need to be very thoughtful about the haphazard usage of anti-cholesterolemic drugs as they may put us at risk for acute tubular necrosis.

Conflict of interest: No conflicts of interest were encountered.

\section{REFERENCES}

1. Henke K: Renal physiology. Dimensions of Critical Care Nursing. 2009; 22 (3):125-132.

2. Luvai A, Mbagaya W, Hall A. Rosuvastatin: A Review of the Pharmacology and Clinical Effectiveness in Cardiovascular Disease. Clin Med Insights Cardiology. 2012; 6: 17-33.

3. Sakaeda T, Kadoyama K, and Okuno Y. Statinassociated muscular and renal adverse events: data mining of the public version of the FDA adverse event reporting system. 2016. $\underline{\text { PLOS }}$ One. ;6 (12): 28124-30.

4. Deichmann R, Lavie C, and Andrews S. Coenzyme Q10 and Statin-Induced
Mitochondrial Dysfunction. Ochsner J. 2010; 10(1):16-21.

5. Abd Elhaleem CZ and Elsayed LA. Coenzyme Q10 Ameliorates Statin-related Myotoxicity: A Biochemical and Histological Study. J. of Pharmacology. 2016; 6 (4): 258-271.

6. Caso G, Kelly P, McNurlan T, and Lawson W. Effect of coenzyme Q10 on myopathic symptoms in patients treated with statins. $A M \quad J$. Cardiology. 2015; 99: 1409-12.

7. Westwood F, Scott R, Marsden A, et al. Rosuvastatin: characterization of induced myopathy in the rat. Toxicology and Pathology. 2016; 36(2):345-52

8. Draeger A, Monastyrskaya K, Mohaupt M, et al. Statin therapy induces ultrastructural damage in skeletal muscle in patients without myalgia. $J$. of Pathology. 2018; 210(1): 94-102.

9. Hegazy R and Hegazy A. Hegazy' Simplified Method of Tissue Processing (Consuming Less Time and Chemicals). Annals of International Medical and Dental Research. 2015; 1(2):57$61 "$.

10. Hayat MR. Basic techniques for transmission electron microscopy. Academic Press Inc., Florida. 2000; p31.

11. Mizuno K, Tanaka M, and Nozaki S. Antifatigue effects of coenzyme Q10 during physical fatigue. Nutrition. 2018; 24(4): 293-299.

12. Rao K, Prasad T, and Manna P. An Overview of Statins as Hypolipidemic Drugs. J. of Pharmaceutical Sciences. 2011; 3 (3): 178-183.

13. Ross H, and Pawlina W. Histology: A text and atlas with correlated cell and molecular biology. Sixth edition. Chapter 20 (2011). Urinary system. Lippincott Williams and Wilkins, New York: 698-723.

14. Ponnuswamy P, Ostermeier E, and Schröttle A. Oxidative Stress and Compartment of Gene Expression Determine Proatherosclerotic Effects of Inducible Nitric Oxide Synthase. AM J. Pathology. 2009; 174 (6): 2400-10.

15. Kostapanos MS, Milionis HJ, and Elisaf MS. Rosuvastatin associated adverse effects and drugdrug interactions in the clinical setting of dyslipidemia. AM J. Cardiovascular Drugs. 2010; 10(1): 11-28.

16. Pisoni R, Wille $\mathrm{K}$, and Tolwani A. The epidemiology of severe acute kidney injury: From BEST to PICARD, in acute kidney injury: New concepts. Nephron Clinical Practice. 2008 ;109 (4): 188-191.

17. Littarru G. Coenzyme Q10 and statins: biochemical and clinical implications. Mitochondrion. 2007; 7 (6): 168-174.

18. Galpern W, and Cudkowicz M. Coenzyme Q10 treatment of neurodegenerative diseases of aging. Mitochondrion. 2007; 7 (6): 146-153.

19. Yabuki A, Suzuki S, and Nishinakagawa D. Sex and strain differences in the brush border and PAS-positive granules and giant bodies of the mouse renal segment cells. Exp. Anim.2011; 50: $59-66$.

20. Thomas M, Brunskill N, and Harris K. Proteinuria induces tubular cell turnover atrophy. A potential mechanism for tubular atrophy. Kidney International. 2019; 55: 890-8.
Anatomy \&

Embryology 
21. Brewer H. Benefit-risk assessment of Rosuvastatin 10 to 40 milligrams. Am $J$. Cardiology. 2013; 92(4):23-29.

22. Zipes D, Zvaifler J, Glassock A et al. Rosuvastatin: An independent analysis of risks and benefits. MedGenMed. 2006; 8 (2): 73-90. 